\title{
TEM, XRD and AFM study of poly(o-ethoxyaniline) films: new evidence for the formation of conducting islands
}

\author{
Fabio L. Leite • William F. Alves • Mirta Mir • \\ Yvonne P. Mascarenhas • Paulo S.P. Herrmann · \\ Luiz H.C. Mattoso • Osvaldo N. Oliveira Jr.
}

Received: 20 November 2007 / Accepted: 15 May 2008 / Published online: 13 June 2008

(c) Springer-Verlag 2008

\begin{abstract}
The existence of conducting islands in polyaniline films has long been proposed in the literature, which would be consistent with conducting mechanisms based on hopping. Obtaining direct evidence of conducting islands, however, is not straightforward. In this paper, conducting islands were visualized in poly(o-ethoxyaniline) (POEA) films prepared at low $\mathrm{pH}$, using Transmission Electron Microscopy (TEM) and atomic force spectroscopy (AFS). The size of the islands varied between 67 and $470 \AA$ for a $\mathrm{pH}=3.0$, with a larger average being obtained with AFS, probably due to the finite size effect of the atomic force microscopy tip. In AFS, the conducting islands were denoted by regions with repulsive forces due to the double-layer forces. On the basis of X-ray diffraction (XRD) patterns for POEA in the powder form, we infer that the conducting islands are crystalline, and therefore a POEA film is believed to consist of conducting islands dispersed in an insulating, amorphous matrix. From conductivity measurements we inferred the charge transport to be governed by a typical quasione dimensional variable range hopping (VRH) mechanism.
\end{abstract}

PACS 73.61.Ph · 72.2.Ee $\cdot$ 68.37.Ps $\cdot$ 61.10.-I $\cdot 71.38 .-\mathrm{k}$

F.L. Leite $(\varangle) \cdot$ W.F. Alves · P.S.P. Herrmann · L.H.C. Mattoso Alan G. MacDiarmid Institute for Innovation and Business and National Nanotechnology Laboratory for Agribusiness (LNNA), Embrapa Agricultural Instrumentation, P.O. Box 741, 13560-970, São Carlos, SP, Brazil

e-mail: leite@cnpdia.embrapa.br

F.L. Leite · Y.P. Mascarenhas · O.N. Oliveira Jr. Institute of Physics of São Carlos, University of São Paulo (USP), P.O. Box 369, 13560-970, São Carlos, SP, Brazil

M. Mir

Department of Exacts Science, Federal University of Alfenas

(UNIFAL), 37130-000, Alfenas, MG, Brazil

\section{Introduction}

Polyaniline (PANI) and its derivatives have been widely studied owing to their stability under ambient conditions and diversity of properties [1, 2]. The highly conducting emeraldine salt of polyaniline (PANI-ES) is formed by protonation of the semiconducting emeraldine base (PANI-EB), without addition or removal of electrons [3]. One important issue in research with polyanilines has been the increased processibility obtained via functionalization of the monomer or the polymeric backbone [4]. This has allowed films to be obtained with various substituted polyanilines, e.g. poly $(o-$ methoxyaniline) (POMA) [5, 6] and poly(o-ethoxyaniline) (POEA) [7, 8]. The degree of protonation and the conductivity vary within the PANI class, which is attributed to differences in conformation of the polymer chains and packing in a film. The mechanisms of charge conduction are still not completely understood, precisely because of the diversity of factors affecting conductivity [9-11]. It is, nevertheless, widely accepted that in the PANI structure the doped molecules are not uniformly distributed, but rather agglomerated into conducting islands [12-14].

The visualization of the conducting islands, however, is far from straightforward. In this paper, we prepared POEA films that were characterized by transmission electron microscopy (TEM) and atomic force spectroscopy (AFS). Strong evidence for conducting islands is obtained, particularly from the analysis of films fabricated at different $\mathrm{pHs}$. These results are compared with X-ray diffraction data for POEA in the powder form. Based on the size distribution of the conducting islands we then discuss the possible mechanisms for charge transport. 


\section{Experimental}

POEA was synthesized according to the procedures described by Mattoso et al. [15]. Layer-by-layer films (LbL) were deposited onto glass substrates. The solution of POEA was prepared in ultra pure water at a concentration of $1 \times 10^{-3} \mathrm{~mol} / \mathrm{L}$ under continuous stirring for $18 \mathrm{~h}$, and the $\mathrm{pH}$ was adjusted to 5.0 by adding $\mathrm{NH}_{4} \mathrm{OH}$. Using this solution 10-layer LbL films were produced by immersion of the substrate into POEA solution for 3 minutes for each layer. Note that LbL films could be produced using only a single material (POEA) because adsorption was favored by $\mathrm{H}$ bonding [16].

$\mathrm{X}$-ray diffraction patterns were collected for POEA samples in the powder form with a Rigaku Rota Flex, on a rotating anode source using a flat-plate Bragg-Brentano geometry, operating with $\mathrm{CuK} \alpha$ radiation, $50 \mathrm{kV}, 100 \mathrm{~mA}$ and equipped with a graphite monochromator. The powder diffractogram was recorded in the range $2 \theta=3-80^{\circ}$ with a step of 0.02. Structural refinement was performed with the Rietveld technique as implemented in the computer program package Fullprof [17]. In this method the overall diffraction profile is calculated as the sum of overlapping peaks whose positions, shape and intensity are defined by the unit cell parameters, zero-point correction, and half-width parameters. All these parameters are refined by the least-squares method to achieve the best agreement between the measured and the calculated profile. Thompson-Cox-Hastings pseudo-Voigt peak profile functions [18] were used to fit the profiles and a linear interpolation between a set background points with refinable heights was used to model the background profile. The initial set with 21 background points was obtained manually using WinPLOTR software [19]. The standard material used for modeling the instrumental resolution was LaB6. The instrumental parameters (Uins, Vins, Wins, Xins, and Yins) were used in the Rietveld refinement. The extraction of the crystallite size was carried out with the Rietveld method using the whole pattern obtained from the refinement procedure. The arbitrary shape of crystallites was simulated using spherical harmonics. The anisotropic size broadening was quantified with the model for triclinic structure and only two independent parameters were refined.

An Atomic Force Microscope TMX 2010 Discoverer, including special cantilevers of $\mathrm{Si}_{3} \mathrm{~N}_{4}(0.032 \mathrm{~N} / \mathrm{m})$, tips with radius $\sim 250 \AA$ and a fluid closed-cell, was used to characterize the 10-bilayer POEA film surfaces. The spring constant of cantilever and radius tip were measured and calculated according to the procedures described by Leite et al. [20]. Transmission electron microscopy (TEM) images were obtained with a Philips CM 120 microscope, for which the samples were prepared by drop casting a POEA solution (ES and EB) onto silicon monoxide/formvar-coated grids (200 mesh). The $d c$ conductivity measurements were carried out on pressed pellets of POEA, doped with $\mathrm{HCl}$, using an apparatus similar to that described earlier [21].

\section{Results and discussion}

Small conducting islands can be seen by comparing the TEM images of the three POEA films in Fig. 1, appearing as black spots in Fig. $1 \mathrm{~b}$ and c, whose size, $D_{i}$, varied between 67 and $440 \AA$ (average size $=192 \pm 121 \AA$ ). The distance, $d_{i}$, between the islands varied between 19

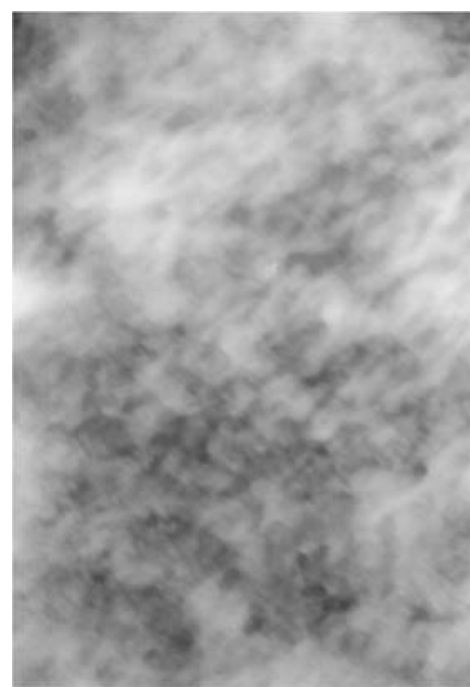

$\mathbf{a}$

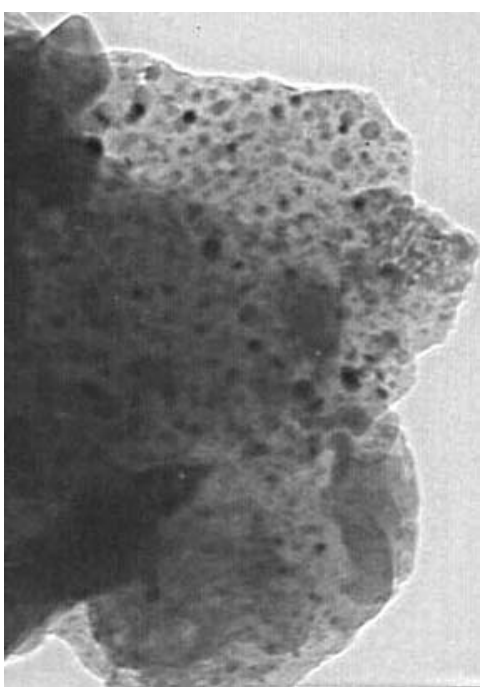

b

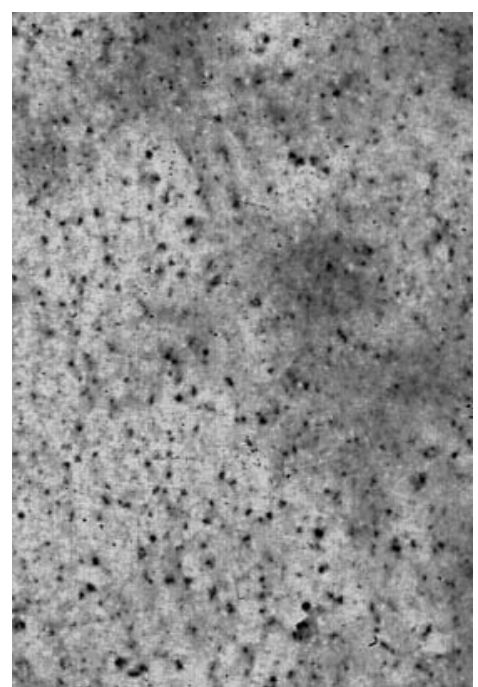

Fig. 1 TEM micrograph of (a) POEA dedoped $(\mathrm{EB})(\mathrm{pH}=10.0)$, and $(\mathbf{b})$ doped $(\mathrm{ES})(\mathrm{pH}=3.0)$ and doped $(\mathrm{ES})(\mathrm{pH}=1.6)$. Magnification $200,000 \mathrm{X}$ 
and $420 \AA$ with an average of $240 \pm 190 \AA$. Similar values were found for polyaniline, viz. $D_{i} \geq 63 \AA$ [22]. These islands also resemble those reported by Lux and Hinrichsen $[23,24]$ for polyaniline, in which $D_{i} \sim 100-300 \AA$ and $d_{i} \sim 100-500 \AA$. The average volume of conducting islands, for a prolate spheroid, is $3 \times 10^{5} \AA^{3}$, which agrees with the values quoted by MacDiarmid et al. [25]. The dark, large areas at the left hand side of the image in Fig. 1b cannot unequivocally be attributed to a conducting phase, since probably the POEA film was too thick in this region. The image in Fig. 1b (POEA-ES), which is characteristic of most conducting samples, also shows irregular channels, with the small black islands immersed in a less dense, insulating matrix. In contrast, the film in Fig. 1a features only large, gray areas, with no dark spots. This was expected since this POEA-EB film was prepared with a high $\mathrm{pH}$ solution ( $\mathrm{pH}>7.0$ ), and therefore was not doped. In subsidiary experiments we noted that the number of conducting islands increased with the degree of doping, which made them closer to each other.

The dark spots representing the conducting islands in Fig. 1 are expected to correspond to crystalline regions, since it is known that upon doping, PANI and its derivatives become conducting and have their crystallinity increased [26]. Indeed, the diffractograms in Fig. 2 shows that POEA-EB is predominantly amorphous, whereas POEA-ES is semi-crystalline. The XRD pattern for the doped, more crystalline POEA was refined, and Fig. $2 \mathrm{~b}$ shows the experimental and calculated patterns in the triclinic phase. The experimental data are denoted by the scatters, and the corresponding calculated profiles are represented by the continuous line. The difference between the observed and calculated results, plotted in blue, is very small. The conventional agreement factors and goodness of fit for the patterns are $R_{\mathrm{p}}=8.71 \% ; R_{\omega \mathrm{p}}=10.2 \%$ and $\mathrm{chi}^{2}=6.11$, respectively. The cell parameters given by Evain et al. [27] were taken as an initial model for cell refinement using the Le Bail method with Fullprof. The POEA structure was refined in a triclinic system with space group $P \overline{1}$. The structural parameters obtained from the refinement are $a=5.412$ (2) $\AA, b=8.845$ (2) $\AA, c=21.612(8), \alpha=84.15(2)^{\circ}, \beta=84.59(2)^{\circ}$, and $\gamma=88.38(1)^{\circ}$ with $Z=2$.

The average crystallite shape obtained from the refinement is shown in Fig. 3, with an average apparent size of $63.03 \AA$ with a standard deviation (anisotropy) of $10.43 \AA$. There is also a smaller size of $43.56 \AA$ in the (001) direction. The average volume of crystallites, for a prolate spheroid, was $V_{\text {crist. }} \sim 6 \times 10^{5} \AA^{3}$, which is within the values for PANI-between 0.7 and $2.6 \times 10^{5} \AA^{3}$ [25, 28]. In addition, this size is consistent with the diameter of the smallest conducting islands observed with TEM. Therefore, it seems that the crystallites in the powder of doped POEA are further aggregated to form larger conducting islands within a film.
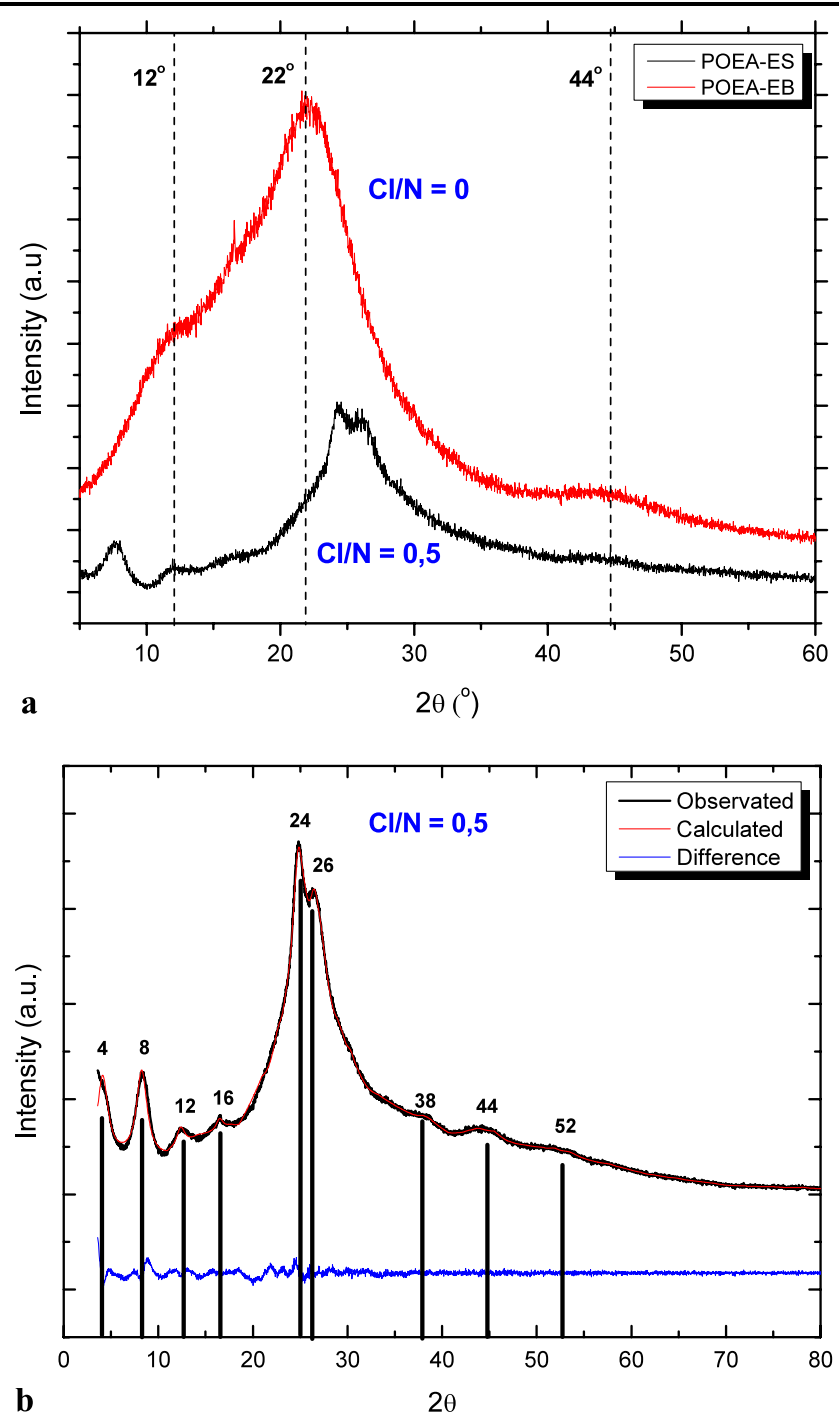

Fig. 2 (a) X-ray diffraction patterns of POEA-EB and (b) Rietveld refinement plot for POEA-ES. The symbols (*) represent the data, while the calculated results appear as the smooth curve. The line at the bottom of the plots is the difference between the observed and calculated results

Another method to interrogate the charge state of conducting polymer samples is atomic force spectroscopy (AFS) [29], but this requires measurements on several points of the sample to avoid adsorption of polymer chains onto the AFM tip. Leite et al. [30] showed that force curves on POEA in contact with aqueous solutions of $\mathrm{pH} \geq 5$ exhibit only attractive forces, as POEA is non-protonated (there is no double-layer, see below). For pHs between 4 and 5 POEA becomes doped, and the repulsive force from the doublelayer counterbalances the van der Waals forces. For $\mathrm{pH}=3$, where POEA is considerably charged, the double-layer force dominates. The reason why the measured forces depend on the surface charge density of the tip and of the film is as follows. The silicon nitride is oxidized at the surface and become charged [31]. These surface charges cause an electric 
field which decreases roughly exponentially with increasing distance from the surface. Thus, when another surface approaches, the double layer is perturbed and the resulting force is known as the double-layer force.

In order to study regions on which double-layer forces dominate, which we refer to as conducting islands, we performed experiments at low $\mathrm{pH}(\mathrm{pH}=3)$. Figure 4 shows an
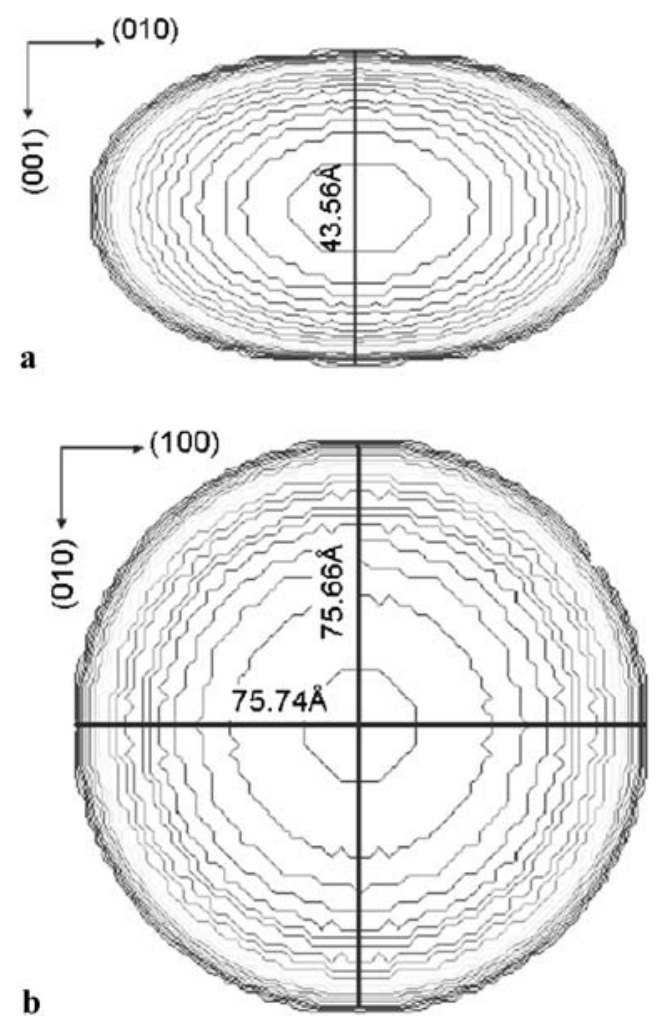

Fig. 3 Visualization of the average crystallite shape obtained from the refinement by reading with GFourier the output binary file generated with fullprof adhesion map with islands of repulsive forces with diameter varying from 100 to $470 \AA$ (average size $=306 \pm 109 \AA$ ) in a matrix with attractive forces. These islands should be made of protonated and crystalline POEA. The result presented by AFS is in surprisingly good agreement with the value (200 to $300 \AA$ ) estimated by Epstein et al. [32] based on conductivity measurements and with those from scanning tunneling spectroscopy (STM) ( 200 to $300 \AA$ ) by Jeon et al. [33]. The average size of the islands is, however, larger than the average obtained with TEM (Fig. 1) and reported for highly oriented PANI-ES films spin-cast from a solution of N-methyl-2-pyrrolidone (NMP) ( $\sim 50 \AA$ ) [34].

A rigorous study involving a combination of techniques, including $d c$ conductivity measurements, can provide useful information on the nature of charge localization, since for most PANI-based materials conductivity is found to show a temperature dependence of the type [35]:

$\sigma=\sigma_{0} \exp \left[-\left(T_{0} / T\right)^{1 / 2}\right]$

which is typical of quasi-one-dimensional variable range hopping (VRH) mechanism between nearest neighbors [36]. Here $T_{0}=16 /\left[\alpha^{-1} N\left(E_{\mathrm{F}}\right) z k_{\mathrm{B}}\right], \alpha^{-1}$ is the decay length of the localized electron state or electron localization length, $N\left(E_{\mathrm{F}}\right)$ is the density of states at the Fermi level, $z$ is the number of nearest neighbor chains (which is assumed to be four for PANI [37]). A fit of the experimental data to equation (1) gives $T_{0}$ values, determined from the slope of the curve.

Figure 5 shows measured conductivity, plotted as log $\sigma$ vs. $T^{-1 / 2}$, with pressed pellets of 2 samples of POEAES, with protonation levels of 0.40 and 0.60 , as obtained from subsidiary X-ray photoelectron spectroscopy (XPS). The straight lines obtained for the $\log \sigma$ vs. $T^{1 / 2}$ data are typical of disordered conductors where the conductivity is
Fig. 4 Map of forces obtained with atomic force spectroscopy showing regions of repulsive (conducting islands) and attractive interactions on POEA films in solution $(\mathrm{pH}=3)$

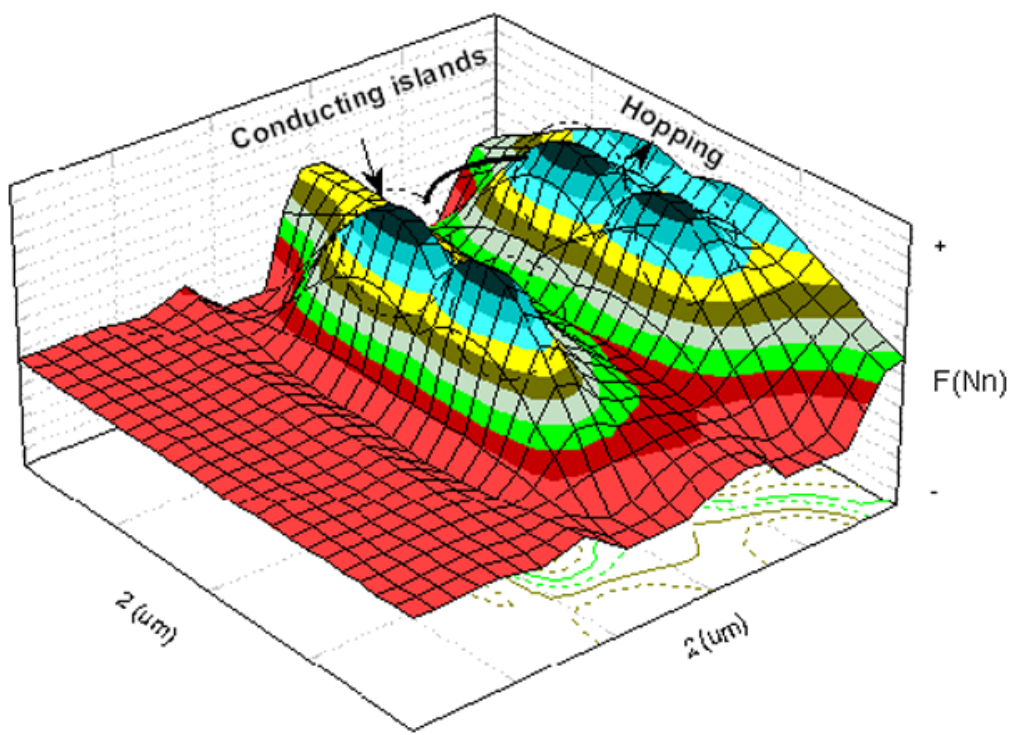




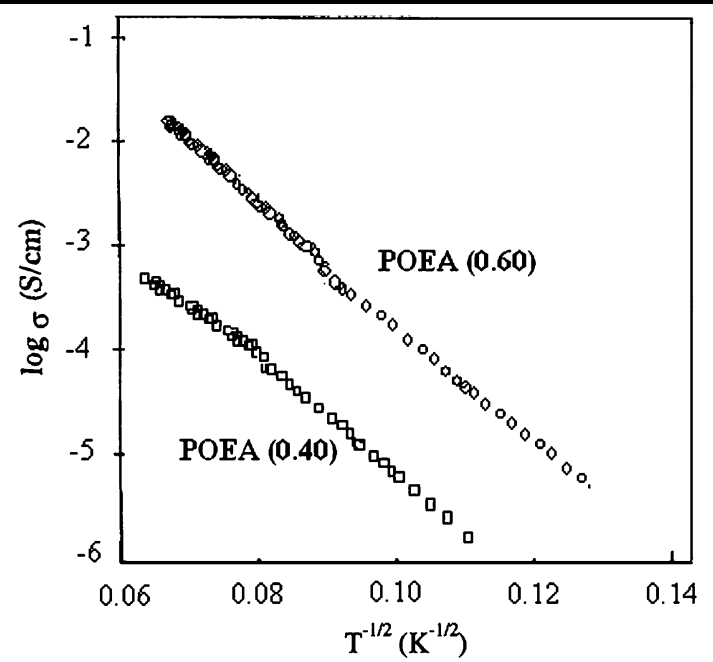

Fig. $5 \log _{10} \sigma$ vs. $T^{-1 / 2}$ for POEA samples in pressed pellets, and doped with $\mathrm{HCl}$

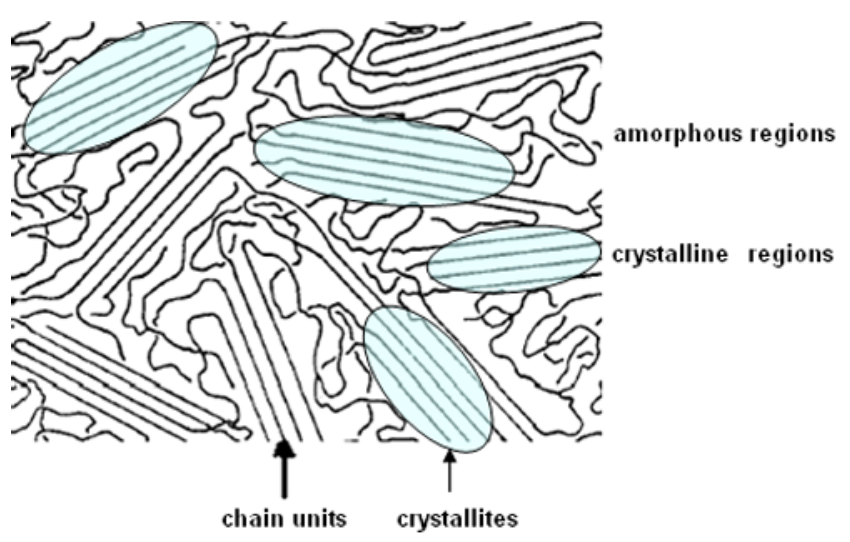

Fig. 6 Schematic representation in 2D of the crystalline and amorphous regions in POEA films

governed by a VRH mechanism in one dimension [38, 39]. Since $T_{0}$ is a measure of the degree of disorder in the system [40], increasing the protonation degree induces further disorder in the packing of chains, thus causing the graph for $x=0.60$ to lie above the other one in Fig. 5, in agreement with the VHR model.

The results from TEM, AFS, XRD, and $d c$ conductivity indicated that POEA films consist of conducting crystallites in an amorphous matrix, which remain unprotonated, as depicted in Fig. 6. The chains in the crystalline regions should be better aligned, which would increase electron delocalization [41]. In doped POEA, though there is a finite density of states of conduction electrons around the Fermi levels, the carriers may be spatially localized due to the structural disorder in the polymers. In the homogeneously disordered regions, the polymer chains are less ordered, thus limiting the overlapping of wave functions [42]. In this case, conduction can only take place through hopping [43, 44], which is the reason why we inserted hopping in the map of Fig. 4.
The rationale above is consistent with the VRH model $[45,46]$, characterized by nearest-neighbor interchain hopping in disordered regions that explains $d c$ conductivity results for POEA (see Fig. 5) and polyanilines [35, 47]. The hypothesis of charge tunneling between metallic islands, in the so-called granular metal model (GMM) [48] cannot be discarded though, at least for the films obtained at $\mathrm{pH}<2$. For in the latter films (see Fig. 1c), the islands are smaller and may be sufficiently close to allow for tunneling. In this case, the domains would be like "metallic grains" embedded in the amorphous, poorly conducting matrix. In the GMM model, charges travel between ordered regions by resonance tunneling through the resonance states in disordered regions [49].

\section{Conclusions}

Using TEM and AFS we showed unequivocally that POEA films are made of conducting islands in an insulating matrix, with the size and number of islands depending on the $\mathrm{pH}$. When the results of XRD for POEA in the powder form are considered, we conclude that the conducting islands consist of crystalline domains, whereas the insulating matrix is amorphous. The islands in a solid film are larger than the crystalline domains seen in XRD patterns, owing to aggregation. This is consistent with a recent morphological study for POEA [50]. Significantly, the average size of the conducting islands was comparable for the AFS and TEM measurements. The larger average for AFS may be associated with the effects from a finite size of the atomic force microscope tip. The findings here lend support to models for charge transport based on hopping, which was confirmed here with $d c$ conductivity measurements. However, the electronic transport could be explained by both VRH and GMM models for films prepared at very low $\mathrm{pH}$, since the islands are smaller and may be sufficiently close to allow for tunneling.

Acknowledgement This work was supported by FAPESP, FINEP and CNPq.

\section{References}

1. Y. Yang, A.J. Heeger, Nature 372, 344 (1994)

2. K. Lee, S. Cho, S.H. Park, A.J. Heeger, C.-W. Lee, S.-H. Lee, Langmuir 441, 65 (2006)

3. A.L. Kon'kin, V.G. Shtyrlin, R.R. Garipov, A.V. Aganov, A.V. Zkharov, Phys. Rev. B 66, 75203 (2002)

4. Y. Wei, R. Hariharan, S.A. Patel, Macromolecules 23, 758 (1990)

5. R.M. Faria, L.H.C. Mattoso, M. Ferreira, O.N. Oliveira, Jr., D. Gonçalves, L.O.S. Bulhões, Thin Solid Films 221, 5 (1992)

6. P.A. Ribeiro, R. Steitz, I.E. Lopis, H. Haas, N.C. Souza, O.N. Oliveira, Jr., M. Raposo, J. Nanosci. Nanotechnol. 6, 1396 (2006) 
7. J.P. Pouget, S.L. Zhao, Z.H. Wang, Z. Oblakowski, A.J. Epstein, S.K. Manohar, J.M. Wiesinger, A.G. MacDiarmid, C.H. Hsu, Synth. Met. 55, 341 (1993)

8. N. Consolin, F.L. Leite, E.R. Carvalho, E.C. Venancio, C.M.R. Vaz, L.H.C. Mattoso, J. Braz. Chem. Soc. 18, 577 (2007)

9. V.N. Prigodin, A.J. Epstein, Physica B 338, 310 (2003)

10. L.-P. Zhou, B. Liu, Z.-Ya. Li, Phys. Lett. A 333, 322 (2004)

11. H.C.F. Martens, J.A. Reedijk, H.B. Brom, D.M. de Leeuw, R. Menon, Phys. Rev. B 63, 073203 (2001)

12. D. Jeon, J. Kim, M.C. Gallagher, R.F. Willis, Science 256, 5064 (1992)

13. J.P. Pouget, M.E. Josefowicz, A.J. Epstein, X. Tang, A.G. MacDiarmid, Macromolecules 24, 779 (1991)

14. R.F. Bianchi, G.F.L. Ferreira, C.M. Lepienski, R.M. Faria, J. Chem. Phys. 110, 4602 (1999)

15. L.H.C. Mattoso, S.K. Manobar, A.G. MacDiarmid, A.J. Epstein, J. Polym. Sci. A 33, 122 (1995)

16. F.L. Leite, L.G. Paterno, C.E. Borato, P.S.P. Herrmann, O.N. Oliveira, Jr., L.H.C. Mattoso, Polymer 46, 12503 (2005)

17. J. Rodriguez-Caravajal, Version 2005 FullProf Program. Rietveld, Profile Matching and Integrated Intensities Refinement of X-ray and/or Neutron Data (powder and/or single-crystal) (Laboratoire Leon Brillouin (CEA-CNRS))

18. P. Thompson, D. Cox, J. Hastings, J. Appl. Crystallogr. 20, 79 (1987)

19. T. Roisnel, J. Rodriguez-Carvajal, in Materials Science Forum, Proceedings of the Seventh European Powder Diffraction Conference (EPDIC 7), ed. by E.R. Delhez, E. Mittenmeijer, pp. 118-123 (2000)

20. F.L. Leite, P.S.P. Herrmann, A.L. Da Roz, F.C. Ferreira, A.A.S. Curvelo, L.H.C. Mattoso, J. Nanosci. Nanotechnol. 6, 2354 (2006)

21. R.M. Faria, R.F. Bianchi, D.T. Balogh, R.J. Ramos, IEEE Trans. Dielectr. Electr. Insul. (EUA) 7, 855 (2000)

22. R. Pelster, G. Nimtz, B. Wessling, Phys. Rev. B 49, 12718 (1994)

23. F. Lux, G. Hinrichsen, V.I. Krinichnyi, I.B. Nazarova, S.D. Cheremisow, M.M. Pohl, Synth. Met. 55, 347 (1993)

24. F. Lux, G. Hinrichsen, M.M. Pohl, J. Appl. Polym. Sci. 32, 1957 (1994)

25. A.J. Epstein, A.G. MacDiarmid, J.P. Pouget, Phys. Rev. Lett. 65, $664(1990)$

26. V.N. Prigodin, A.N. Samukhin, A.J. Epstein, Synth. Met. 141, 155 (2004)

27. M. Evain, S. Quillard, B. Corraze, W. Wang, A.G. MacDiarmid, Acta Crystallogr. E 58, 0343 (2002)
28. J. Joo, S.M. Long, J.P. Pouget, E.J. Oh, A.G. MacDiarmid, A.J. Epstein, Phys. Rev. B 57, 9567 (1998)

29. F.L. Leite, P.S.P. Herrmann, J. Adhes. Sci. Technol. 19, 365 (2005)

30. F.L. Leite, C.E. Borato, W.T.L. da Silva, P.S.P. Herrmann, O.N. Oliveira, Jr., L.H.C. Mattoso, Microsc. Microanal. 13, 304 (2007)

31. H.-J. Butt, Biophys. J. 60, 1438 (1991)

32. F. Zuo, M. Angelopoulos, A.G. MacDiarmid, A.J. Epstein, Phys. Rev. B 36, 3475 (1987)

33. D. Jeon, J. Kim, M.C. Gallagher, R.F. Willis, Science 256, 1662 (1992)

34. Z.H. Wang, C. Li, E.M. Scherr, A.G. MacDiarmid, A.J. Esptein, Phys. Rev. Lett. 66, 1745 (1991)

35. A. Raghunathan, P.K. Kahol, B.J. McCormick, Solid State Commun. 108, 817 (1998)

36. E.P. Nakhmedov, V.N. Prigodin, A.N. Samukhin, Sov. Phys. Solid State 31, 368 (1989)

37. Z.H. Wang, A. Ray, A.G. MacDiarmid, A.J. Epstein, Phys. Rev. B 43, 4373 (1991)

38. A. Raghunathan, T.S. Natarajan, G. Rangarajan, S.K. Dhawan, D.C. Trivedi, Phys. Rev. B 47, 13189 (1993)

39. M. Gosh, A.K. Meikap, S.K. Chattopadhyay, S. Chatterjee, J. Phys. Chem. Solids 62, 475 (2001)

40. A. Raghunathan, P.K. Kahol, B.J. McCormick, Synth. Met. 100, 205 (1999)

41. G. Zotti, M.C. Gallazzi, G. Zerbi, S.V. Meille, Synth. Met. 73, 217 (1995)

42. A.B. Kaiser, Rep. Prog. Phys. 64, 1 (2001)

43. R.S. Kohlman, J. Joo, A.J. Epstein, in Physical Properties of Polymers Hand Book, ed. by J.E. Mark (Am. Inst. Phys., New York, 1996), p. 453

44. J.S. Nogueira, L.H.C. Mattoso, C.M. Lepienski, R.M. Faria, Synth. Met. 69, 259 (1995)

45. N.F. Mott, M. Kaveh, Adv. Phys. 34, 329 (1985)

46. Z.H. Wang, E.M. Scherr, A.G. MacDiarmid, A.J. Epstein, Phys. Rev. B 45, 4190 (1992)

47. A. Raghunathan, P.K. Kahol, B.J. McCormick, Synth. Met. 101, $732(1999)$

48. P. Sheng, B. Abeles, Y. Arie, Phys. Rev. Lett. 31, 44 (1973)

49. V.N. Prigodin, A.J. Epstein, Synth. Met. 125, 43 (2002)

50. F.L. Leite, M. Oliveira Neto, L.G. Paterno, M.R.M. Ballestero, I. Polikarpov, Y.P. Mascarenhas, P.S.P. Herrmann, L.H.C. Mattoso, O.N. Oliveira, Jr., J. Colloid Interface Sci. 316, 376 (2007) 